\title{
6. Contre l'évidence spatiale ou les aléas de l'hypothèse localiste des prépositions
}

\section{Pierre Péroz}

\section{(2) OpenEdition}

12 Journals

Édition électronique

URL : https://journals.openedition.org/ml/576

DOI : $10.4000 / \mathrm{ml} .576$

ISSN : 2274-0511

Éditeur

Association Modèles linguistiques

Édition imprimée

Date de publication : 1 juillet 2006

Pagination : 67-80

Référence électronique

Pierre Péroz, « 6 . Contre l'évidence spatiale ou les aléas de l'hypothèse localiste des prépositions », Modèles linguistiques [En ligne], 54 | 2006, mis en ligne le 01 octobre 2015, consulté le 01 juillet 2021. URL : http://journals.openedition.org/ml/576 ; DOI : https://doi.org/10.4000/ml.576

Ce document a été généré automatiquement le 1 juillet 2021

(C) Modèles Linguistiques 


\title{
6. Contre l'évidence spatiale ou les aléas de l'hypothèse localiste des prépositions
}

\author{
Pierre Péroz
}

1 Que l'espace soit le mode normal de nos représentations lorsqu'il s'agit de répondre à une demande de figuration explicite, cela est à peu près indiscutable. Si l'on demande à quelqu'un ce qu'est un livre, ce qu'est un accident, ce qu'est une patience, ou même ce que signifie voter contre, il y a de fortes chances que le sujet se représente visuellement les choses avant de répondre. De là à en tirer la conclusion que ce type de représentation structure selon des modalités homothétiques la construction du sens dans le langage, il y a un pas. Ce pas est franchi par les tenants de «l'hypothèse localiste » ainsi que la nomme C. Vandeloise (2005: 121) qui s'en fait le défenseur dans l'article ${ }^{1}$ que je vais étudier ici.

2 C. Vandeloise ne définit pas clairement cette expression dans l'un ou l'autre de ses articles sur contre (2003 et 2005). Pour en avoir une approximation, il faut nous reporter à son ouvrage sur L'espace en français (1986) dans lequel il en donne les principes, sans utiliser l'expression qui, à l'époque, n'existait pas. Rappelons les brièvement. Il montre d'abord que l'analyse des emplois spatiaux des prépositions comme devant, derrière, sur, dans, etc. ne peut se faire dans le cadre de la géométrie euclidienne; il est en effet nécessaire d'introduire dans l'analyse la notion d'orientation, laquelle suppose généralement la présence d'un observateur absent de la géométrie en question. Elle ne peut pas non plus se faire dans le cadre strict de la logique des prédicats, celle-ci ignorant généralement la relation asymétrique entre les deux termes mis en relation par la préposition que C. Vandeloise va nommer désormais «cible » et "site ». Il propose donc une description des dites prépositions (1986:22) qui mobilise «des concepts fonctionnels liés à la connaissance extralinguistique de l'espace que partagent les locuteurs d'une même langue ». Il dégage cinq groupes de traits universels [...] qui constituent des "primitifs indéfinissables en fonction desquels [il effectue] la description des expressions spatiales ». Ce sont : 1 . la forme du corps humain - en tant 
qu'il fournit les éléments nécessaires à l'orientation - et la notion "d'interaction canonique » («la position selon laquelle deux interlocuteurs se parlent, face à face, à une courte distance l'un de l'autre ») ; 2 . la physique naïve, relative à notre perception naturelle des relations entretenues par les entités matérielles dans le monde ${ }^{2} ; 3$. l'accès à la perception, correspondant à l'organisation naturelle des entités dans notre champ visuel; 4. la rencontre potentielle, qui correspond au fait que "toutes nos actions peuvent s'exprimer en termes de rencontre» («toute rencontre implique un mouvement, l'inverse n'est pas vrai») et 5. l'orientation générale et l'orientation latérale. D'où le caractère extrêmement référentialiste ou visuel de ses analyses. Pour décrire La tasse est sur la table, C. Vandeloise fait un dessin ${ }^{3}$. Du coup, dans la mesure où il s'en tient au domaine spatial, ses analyses ont une évidence indiscutable.

3 Mais «l'hypothèse localiste " va au-delà. Il s'agit non seulement de dire que, dans le langage, l'espace est notre premier domaine de représentation mais que les autres domaines en dérivent. Étudier les emplois spatiaux, c'est donc étudier les emplois qui fondent tous les autres. C. Vandeloise souscrit maintenant à cette hypothèse, il le dit au début de son article de 2005 : "Je crois que le localisme constitue une hypothèse fructueuse pour expliquer le développement de plusieurs prépositions spatiales ${ }^{4}$ comme sur et dans » (p. 121). Il va donc tenter, en s'inscrivant dans cette logique : « de dériver les usages axiologiques de contre des mouvements impliqués par ses usages spatiaux » (p. 121) et, dans un deuxième temps, de rassembler ce qu'il nomme les règles d'usage de la préposition sous le concept unificateur de « rencontre atypique ». Comme on le voit, de 1986 à 2005, C. Vandeloise, reste aussi fidèle à lui-même - on aura reconnu dans la rencontre atypique un écho inversé mais très clair de la rencontre canonique il conclut son introduction (p. 123) en des termes qui sont ceux de 1986: «Si l'espace contribue ainsi à unifier l'usage de contre, ce n'est pas à travers sa structure géométrique ou topologique mais par l'usage que nous en faisons ».

Son article comporte cinq parties inégales : la première, assez brève (pp. 121-125), est une introduction générale sur la notion de " rencontre », la seconde (pp. 125-133) porte sur les emplois spatiaux de contre et la troisième (pp. 134-139) porte sur les emplois axiologiques, la quatrième (pp. 139143) se veut une comparaison entre ces deux types d'emplois et la cinquième partie traite en conclusion de la "rencontre atypique » (pp. 1431-45).

5 Nous allons simplement suivre les différentes étapes de son raisonnement pour montrer les questions que cette lecture soulève et qui nous font remettre en cause les fondements de sa démarche.

\section{L'opposition et la rencontre}

Dans un premier temps, C. Vandeloise passe en revue quelques concepts utilisés par d'autres mais qui lui paraissent moins pertinents que celui de « rencontre $»^{5}$.

7 Il écarte d'abord la notion d'« opposition », souvent retenue comme fédératrice par les dictionnaires (TLF cité par l'auteur), parce que, si les entités mobilisées dans les emplois spatiaux peuvent être en mouvement et dans des directions contraires, elles ne sont pas «en opposition"; l'opposition étant réservée selon lui aux emplois axiologiques. Mais il écarte aussi une description qui serait basée sur la rencontre de "deux mouvements opposés » parce que (p. 124), ce type suppose un troisième terme, « un point de référence par rapport auquel les deux mouvements sont évalués » tandis 
que «les rencontres les plus élémentaires, essentielles pour définir l'usage de contre, n'impliquent en revanche que deux $\left(\mathrm{cnqs}^{6}\right)$ éléments". C'est donc le terme de « rencontre » que $\mathrm{C}$. Vandeloise retient pour décrire les emplois spatiaux de contre.

Il rappelle qu'il a défini (p. 124) dans C. Vandeloise (2003) : « les usages spatiaux de la préposition contre par la rencontre élémentaire entre un élément mobile et un élément immobile ». On peut juger la définition un peu courte (elle vaut aussi bien par exemple pour La tasse sur la table, Le chien dans la niche, Le tableau au mur), mais la suite va éclairer son propos.

\section{Les usages spatiaux de contre}

9 En fait, la définition de la notion de "rencontre » est donnée en extension, par la construction d'une typologie, elle-même basée sur les critères de mobilité et d'orientation des entités mises en relation par contre. L'auteur propose un tableau (p. 126), reproduit ci-dessous, qui tend à montrer que les emplois spatiaux de la préposition contre peuvent se ramener à l'une ou l'autre des quatre formes de " rencontre »; la « rencontre " se définissant elle-même comme la notion qui se trouve actualisée à travers les quatre types d'emplois spatiaux de contre. La circularité de la définition n'est pas gênante si l'on sait ce que sont les emplois « spatiaux » de contre.

\begin{tabular}{cccc} 
Entité-sujet & Site & \multicolumn{1}{c}{ Section } \\
Mobile & Immobile & $\mathbf{C}$ & section 2.1 \\
Immobile & Mobile & $\mathbf{-}$ & section 2.2 \\
Mobile & Mobile & $\mathbf{\leftarrow}$ & section 2.3 \\
Immobile & Immobile & $\mathbf{0 0}$ & section 2.4
\end{tabular}

Le tableau semble répondre assez clairement à la question dans la colonne qui est dépourvue de titre. Mais est-ce aussi clair que cela? Pour le savoir, il faut bien saisir la logique du tableau. La schématisation des différents types de rencontre pourrait laisser penser que le point de départ du tableau est spatial. En réalité ce n'est pas le cas, ce sont les emplois eux-mêmes qui sont à l'origine du tableau. Si, en effet, on se posait la question de la rencontre dans le domaine spatial, indépendamment de son expression par contre, deux lignes au moins du tableau seraient à supprimer, la deuxième équivalant à la première et la dernière qu'on peut difficilement décrire comme une « rencontre ». Mais, une fois que l'origine linguistique du tableau est établie, il faut comprendre que sa logique interne, elle, est de nature spatiale. C'est-à-dire qu'on ne passe pas d'une ligne à l'autre sur la base de critères linguistiques, mais sur la base de critères spatiaux. On passe d'une ligne à l'autre parce que l'énoncé décrit un mouvement différent dans l'espace. C. Vandeloise lui-même ne manque pas de souligner ${ }^{8}$ que l'équilibre du tableau ne vaut que sur le plan spatial; sur le plan linguistique, il est nettement déséquilibré. La première ligne regroupant la plupart des énoncés tandis que la dernière ne donne lieu qu'à un seul type d'énoncé, relativement contraint, comme L'école est contre la mairie'. Mais l'auteur n'a pas de raison de présenter cela comme un élément susceptible de remettre en cause sa démonstration qui repose sur la complémentarité indiscutable des deux premières colonnes. Indiscutable. Jusqu'à 
quel point? Voici les principaux énoncés sur lesquels C. Vandeloise fait porter son analyse.

\begin{tabular}{|c|c|c|c|l|}
\hline Entité sujet & \multicolumn{1}{|c|}{ Site } & \multicolumn{2}{|c|}{ Section } & \multicolumn{1}{c|}{ Exemples - types } \\
\hline \multirow{2}{*}{ Mobile } & \multirow{2}{*}{ Immobile } & 21 & 21,1 & $\begin{array}{l}\text { (9) Ferdinand jette la Pierre } \\
\text { (contre le mur) } \\
\text { (10) Ferdinand bute contre }\end{array}$ \\
\cline { 3 - 5 } & 21,2 & $\begin{array}{l}\text { une brique } \\
\text { (11) L'échelle est contre le } \\
\text { mur }\end{array}$ \\
\hline
\end{tabular}

\begin{tabular}{|c|c|c|l|}
\hline Immobile & Mobile & 22 & $\begin{array}{l}(21) \text { Le mur protége le jardin } \\
\text { cowte les voleurs }\end{array}$ \\
\hline Mobile & Mobile & 23 & $\begin{array}{l}\text { (31) Ferdinand nage contre le } \\
\text { courant }\end{array}$ \\
\hline Immobile & Mobile & 24 & $\begin{array}{l}(44) \text { Les soldats se serrent contre } \\
\text { le feu. Ferdinand parle contre } \\
(45) \text { Fere } \\
\text { 1oreille de Churles } \\
(46) \text { L'ecole est (tout) contre la } \\
\text { mairie }\end{array}$ \\
\hline
\end{tabular}

Trois choix font question :

- pourquoi classer dans la section $21^{10}$ (rencontre entre une entité-sujet mobile et un site immobile) des énoncés dont l'entité-sujet est immobile (comme L'échelle est contre le mur), alors qu'il existe déjà une section (24) supposée regrouper les énoncés dont les deux entités sont immobiles (comme L'école est contre la mairie) ? En (9) et (10), ce sont les verbes buter et jeter qui indiquent que la cible est mobile mais on ne voit pas pourquoi l'échelle en (11) est dite «mobile», puisque la situation décrite est statique (par opposition par exemple à L'échelle glisse contre le mur.

- pourquoi classer comme spatiaux des énoncés que d'autres auteurs comme A. Borillo (2003) ou P. Dendale (2003), qui sont à peu de choses près sur les mêmes bases théoriques, considèrent comme axiologiques, en l'occurrence des énoncés avec les verbes (se) défendre ou (se) protéger comme 21 : Le mur protège le jardin contre les voleurs?

- pourquoi classer des énoncés du type L'école est contre la mairie dans ce tableau dont le concept unificateur est la « rencontre»?

Voyons donc plus précisément cela.

\section{1. Échelle mobile}

Dans la section 21.3: "Rencontre entre une entité-sujet mobile et un site immobile ", les énoncés comme L'échelle est contre le mur font problème puisque les deux entités sont immobiles. Il faut donc ajouter un codicille au tableau pour justifier ce classement. Ce que fait C. Vandeloise de la façon suivante (p. 128) : «Ces rencontres s'effectuent entre un site immobile et une entité qui bougerait (cnqs) sans la présence du site qui lui sert de point d'appui. Il s'agit donc d'une rencontre entre une entité potentiellement mobile (cnqs) et un site immobile».

14 Le problème est alors celui du critère retenu : qu'est-ce qu'une entité « potentiellement mobile »? Si l'on s'en tient à ce qui vient d'être précisé, on devrait ranger dans la même sous-classe, des énoncés comme :

15 L'affiche est collée contre le mur.

Il l'a plaquée contre le mur. 

21.1. Il lui faut donc ajouter un nouveau codicille (p. 128) qui est que dans les énoncés du type 21.1 « Les forces impliquées sont des forces imprimées ou auto imprimées » (ce qui inclut les deux énoncés que nous venons de donner), tandis que, pour les exemples du type 21.3. (L'échelle est contre le mur), ce sont « des forces naturelles provoquées par la pesanteur et la gravité ». Cela ne résout pas vraiment le problème sur le fond. On pourrait en effet faire remarquer que la pesanteur maintient l'échelle, mais qu'elle ne se trouve là que parce qu'on l'y a mise et donc par le biais d'une force imprimée. Ce, qui en toute logique, devrait faire ranger l'énoncé dans la sous-classe 21.1. Mais n'allons pas plus loin et voyons maintenant la deuxième question.

\subsection{Bloqué quelque part dans l'espace-temps}

L'attention du lecteur en effet ne peut manquer d'être attirée par les énoncés supposés illustrer la deuxième ligne du tableau (section 22). On comprend bien que pour l'équilibre de la présentation - dans le cadre de l'hypothèse localiste - il vaut mieux trouver des exemples de cette orientation complémentaire de celle de la section 21 . Ce sont donc des énoncés comme, Le mur protège le jardin contre les voleurs qui sont donnés. C. Vandeloise ne manque pas de noter que le site de la relation n'est pas directement l'entité nominale, mais le processus ou l'activité dans lesquels l'entité est impliquée ${ }^{11}$. Mais, comment dans ce cas, justifier le classement spatial de ces énoncés? C. Vandeloise concède que «ces phrases suggèrent un lien entre les usages spatiaux de contre et les usages axiologiques ». Il serait pourtant nécessaire d'aller plus loin. On a déjà signalé que le caractère spatial de ces énoncés était loin d'être admis par tous les auteurs. Un bref essai de construction d'énoncés proches devrait permettre de le vérifier :

Le mur protège le jardin contre les voleurs.

Le bateau s'abrite contre la tempête.

Les marins se défendent contre la tempête.

Les ouvriers se défendent contre le patronat.

Il s'agit de protéger notre économie contre des visées malveillantes.

Il s'agit de protéger le droit de la propriété intellectuelle contre un lâchage inacceptable du gouvernement.

Il faut protéger l'économie nationale contre les risques d'une désinflation brutale.

On me dira alors que c'est une question de lexique. Pourquoi pas? Mais dans ce cas, cela suppose qu'on définisse le type d'entités mobilisées, ce que ne fait pas l'auteur, qui se borne à donner une condition nécessaire mais non suffisante de la mise en place de ces emplois en constatant (p. 129) que «Ce type d'usages spatiaux de contre n'apparaît que derrière quelques verbes, comme défendre et protéger ». Mais il serait faux de croire qu'il s'agit d'un simple oubli. La démarche retenue par l'auteur est en quelque sorte rétive à la prise en compte de cette dimension lexicale. Il lui faudrait pour cela mobiliser des critères proprement linguistiques dont on a vu au début qu'ils ne faisaient pas partie de la panoplie théorique mise en avant. Cela est patent lorsqu'il est confronté à des énoncés qui ne correspondent pas au schéma établi et qu'il lui faut prendre en compte la dimension lexicale. On pourra en juger sur le commentaire qu'il fait de l'exemple suivant (p. 142) :

(86) Ferdinand bute toujours contre la même idée. 
«Les sites de contre spatial sont normalement concrets. Ce n'est pas le cas dans la phrase (86). Même si les sites sont abstraits, l'usage de contre dans cette phrase n'est pas axiologique. Il s'agit plutôt d'un emploi métaphorique (cnqs) de idée, qui est interprété comme un obstacle physique. »

21 Ce commentaire appelle deux remarques. La première est que, s'il fallait identifier la métaphore, il y a de fortes chances que ce soit le verbe qui soit dit métaphorique et non le nom idée, employé dans son acception habituelle. La seconde est que le surgissement, à deux pages de la fin de l'article, d'emplois spatiaux de contre avec un site " abstrait ", selon le mot employé par C. Vandeloise, ouvre brutalement des perspectives que l'analyse en termes de représentation spatiale ne laissaient pas soupçonner, mais que l'auteur ne va pas envisager.

C. Vandeloise n'explore donc pas cet aspect des choses. Il s'en tient pour son analyse aux exemples les plus clairement spatiaux pour établir (p.132) à partir des énoncés des sections 21 et 22 qu'une des règles d'usage de la préposition contre se formule ainsi : Cs : $x V(z)$ contre y si une des entités arrête (ou freine) l'autre.

Comme on vient de le voir, cette règle ne rend pas clairement compte des énoncés avec les verbes défendre ou protéger, qui ne mettent pas en jeu une entité mais un processus associé qui peut être arrêté ou freiné mais alors, non pas seulement sur le plan spatial, mais sur le plan spatio-temporel et dans certains cas sur le plan temporel.

\subsection{Faux mouvement}

Il y a enfin la question de " la rencontre de deux entités immobiles ». C'est, bien sûr, la quatrième ligne du tableau qui pose le plus de problèmes. Comment justifier sa présence dans un tableau fondé sur la notion de « rencontre »? C. Vandeloise va faire valoir deux types d'arguments que nous allons discuter. Le premier est qu'il existe d'autres énoncés que le seul L'école est contre la mairie; on aurait donc une classe substantielle d'énoncés. Le second est que cet exemple, certes délicat, pourra s'expliquer par la notion de « rencontre atypique ».

\subsubsection{Une classe substantielle d'énoncés}

Il existe donc, selon lui, d'autres énoncés mettant en œuvre la même configuration spatiale. Soit donc (p. 132) : (44) Les soldats se serrent contre le feu; pour justifier le classement de cet énoncé dans la sous-classe 24 (rencontre entre deux entités immobiles), C. Vandeloise fait remarquer (citant Dendale 2002) que les soldats ne touchent pas le feu - pas plus que ne le font la mairie et l'église - alors que "tous les usages de contre jusqu'ici traités impliquaient le contact». On peut tout de même s'étonner qu'une caractéristique aussi importante ${ }^{12}$ - le contact - n'ait pas été déjà soulignée, alors que l'auteur a développé deux sous-règles rien que pour le plutôt rare L'échelle est contre le mur. Mais on peut aussi se dire que le critère surgi brusquement est ad hoc. Ad hoc, parce que la notion de contact, n'est pas immédiatement acceptable dans un énoncé qui relèverait de la section 22 comme :

Vous avez une arme? Ben oui, on se protège comme on peut contre les voleurs.

De même qu'il faudrait redéfinir cette notion pour rendre compte des exemples dans lesquels on a un site "abstrait» selon les termes de C. Vandeloise, comme le (86) déjà cité. Mais on peut laisser là la question du contact, déjà très discutée par le même $\mathrm{P}$. 
Dendale (2002), pour revenir au fond de l'affaire qui consiste à dire que dans l'énoncé (44), les deux entités sont immobiles. Car enfin, le verbe se serrent marque assez clairement le caractère dynamique du procès. Les soldats ne se serrent pas les uns contre les autres mais contre le feu. C. Vandeloise ajoute lui-même (p. 132) que « Il est cependant vrai que le feu empêche les soldats de s'approcher (cnqs) plus près ». On ne peut mieux dire qu'il s'agit d'entités mobiles. D'ailleurs $C$. Vandeloise finit lui-même par dire que la règle d'usage qui s'applique aux énoncés dynamiques s'applique aussi dans ce cas ${ }^{14}$. Voilà la classe des énoncés de la section 24 réduite d'un tiers. L'auteur propose ensuite la séquence (45) ainsi formulée : Ferdinand parle contre l'oreille de Charles. On ne trouve pas de telles attestations, mais plutôt des énoncés du type : Ferdinand parle a l'oreille de Jacques. Ou encore des énoncés comme les suivants, qui impliquent beaucoup plus clairement que la « cible » est mobile :

Enfin, au niveau individuel, il faut éviter de coller le portable contre l'oreille (c'est facile en phase de recherche et d'appel) ; l'usage d'un (sites.persoo.net/ciarteme)

Les combinés [...] sont préférables à ceux qui obligent l'utilisateur à appliquer l'antenne contre l'oreille...

31 Il colla sa bouche contre l'oreille du vieillard et, tandis que ce dernier (Henri Queffélec Un recteur de l'île de Sein, 1944, pages 17-18, dans Frantext)

[...] il me pressait dans les mains, un grand coquillage des mers du Sud qu'il m'appliquait contre l'oreille et je finissais tout de même par m'endormir (Blaise Cendrars, Bourlinguer, 1948, pages 28-29, dans Frantext)

C'est-à-dire des énoncés qui relèvent de la sous-classe 21 (mouvement freiné ou arrêté par le site). D'ailleurs, C. Vandeloise finit par écrire (p. 133) que l'énoncé (45) relève finalement de la règle " $\mathrm{Cs}$ ", puisque Ferdinand comme les soldats « sont capables de se déplacer ». Voilà la classe 24 réduite des deux tiers. C'est-à-dire qu'on est ramené au seul exemple reformulé ainsi par C. Vandeloise : (46) L'école est (tout) contre la mairie, pour lequel il lui faut toujours justifier l'idée d'une rencontre entre deux entités immobiles ${ }^{15}$. C. Vandeloise, faisant constater le caractère marginal de l'énoncé conclut qu'il n'est pas « parfaitement couvert par la règle d'usage $C s$ » qui vaut pour les entités mobiles et il annonce: "Je tenterai de justifier cet usage par la notion de rencontre atypique à la section 5 . ». On doit à la vérité de dire qu'il n'est pas fait mention de ce type d'énoncé dans la dite section 5 qui définit ce qu'est une rencontre atypique. Finalement, sur quoi peut-on s'appuyer? Qu'est-ce qui reste à l'issue de ce parcours mouvementé?

\subsection{Conclusion d'étape}

L'équilibre schématique du tableau initial est mis à mal. Sur l'ensemble des énoncés qu'intuitivement on peut classer comme spatiaux, seuls ceux de la section 21 et ceux de la section 23 répondent correctement aux règles d'usage rédigées par l'auteur. Les autres font problème, soit qu'ils relèvent autant du domaine temporel que du domaine spatial, soit qu'ils soient rétifs à toute description en termes de mouvement, alors que le mouvement est le fondement des règles de description. J'en tirerai deux conclusions pour la suite du propos relativement aux hypothèses de départ.

- La première, qui est à proprement parler celle de l'hypothèse localiste, qui voudrait que les emplois autres que spatiaux sont génétiquement dérivés des emplois spatiaux et puissent 
donc être décrits dans les mêmes termes, moyennant des adaptations de circonstances, est sérieusement mise à mal par les constats que nous venons de faire.

- La seconde, défendue depuis 1986 par l'auteur, selon laquelle un concept global - ici celui de « rencontre atypique » - basé sur l'espace ou plutôt (p. 123) «l'usage que nous en faisons » devrait être suffisant pour rendre compte de tous les emplois de la préposition, se trouve à priori fragilisée. nos représentations, suppose qu'on soit à même de faire la part de ce qui est spatial et de ce qui n'est l'est pas. On a vu que ce n'était pas si simple. La définition différentielle des emplois axiologiques s'impose donc à $\mathrm{C}$. Vandeloise comme un élément essentiel de son argumentation. Voyons ce qu'il propose. D'une part, il les distingue sur le plan syntaxique, lexical et sémantique et, d'autre part, il va proposer pour eux des règles d'usage spécifique.

C. Vandeloise fonde d'abord cette distinction (p. 134) sur «l'existence d'un antonyme spécifique [aux axiologiques], la préposition pour». Puis il distingue deux types d'axiologiques : les énoncés à verbe être du type (47) Ferdinand est contre la guerre et les énoncés qui comportent un verbe d'expression comme dans (71) Ferdinand chante une chanson contre la guerre. Les premiers se distinguent des emplois spatiaux par le fait qu'on peut effacer ou déplacer le site de la relation comme dans (49) La guerre, Ferdinand est contre ${ }^{16}$.

\section{C.Vandeloise introduit de la manière suivante (p. 137) :}

«Comme je l'ai fait pour la description des prépositions dans et sur par les relations extra-linguistiques du contenant au contenu (relation $\mathrm{C} / \mathrm{c}$ et du porteur au porté (relation $\mathrm{P} / \mathrm{p}$ respectivement (Vandeloise 1986), j'utiliserai le verbe satisfaire dans la définition de contre pour introduire une notion extra-linguistique, à savoir la notion de satisfaction $^{17}$. Les relations $\mathrm{C} / \mathrm{c}$ et $\mathrm{P} / \mathrm{p}$ sont des notions essentielles pour notre survie dans le monde. A ce titre, elles font partie d'un inventaire limité de notions qui permettent de mettre en forme notre expérience du monde. La notion de satisfaction pourrait être jugée plus primitive encore puisqu'elle est l'arbitre qui classe toutes nos actions en réussies ou ratées: mettre le café dans la tasse est satisfaisant mais le mettre hors de la tasse ne l'est pas ; mettre le livre sur la table est satisfaisant mais le mettre à côté ne l'est pas, etc. Ce qui détermine notre satisfaction est un ensemble de principes qui partent des besoins biologiques les plus fondamentaux pour aller jusqu'à nos caprices individuels les plus personnels en passant par les principes généraux établis par chaque société. On peut donc proposer la règle provisoire $\mathrm{Ca}^{\prime}[. .$.$] , si on admet que, dans cette règle, le verbe$ satisfait désigne une notion extra-linguistique primitive de satisfaction :

$\mathrm{Ca}^{\prime}$ : $x$ est pour / contre y si un processus déterminé par $y$ satisfait / ne satisfait pas $x$

Le processus peut être un choix ou un déroulement et ne garantit pas l'accès à y. "

Je vais donc reprendre ces différents points qui délimitent sur différents plans la spécificité des emplois axiologiques. 
Il est clair qu'une délimitation strictement lexicale de la sous-classe appelle des contreexemples, qui, bien sûr, ne manquent pas. Que faire d'énoncés dans lesquels on ne trouve ni verbe d'état ni verbe d'expression comme dans :

Le sort s'acharne contre lui.

Ils ont eu recours à des procédés contre nature.

La règle d'usage mobilise, elle, deux notions différentes, celle de " processus » et celle de « satisfaction».

- La première est de nature linguistique. C. Vandeloise observe que, dans les emplois axiologiques, le site de la relation n'est pas une entité, mais un processus ou un déroulement dans lequel le site $y$ se trouve impliqué. Que n'a-t-il fait cette observation plus tôt ? Il aurait intégré les énoncés du type se protéger contre, qui vérifient, comme on l'a vu, ce critère, puisqu'il s'agit d'énoncés de type axiologique. Mais on a vu aussi que cela aurait mis à mal l'équilibre du tableau spatial...

- La seconde est de nature extra-linguistique, d'après $C$. Vandeloise. Il ne s'agirait pas de la satisfaction telle qu'on peut l'envisager à partir des emplois du mot «satisfaction» (ou satisfait?), mais telle qu'on peut l'éprouver, les deux prépositions pour et contre s'opposant justement sur la base de la satisfaction de x. Je n'épiloguerai pas sur la difficulté de définir en termes opératoires ce qu'est une satisfaction extralinguistique. Admettons un instant que cela soit possible ; comment pourrait-on rendre compte de l'énoncé que je viens de donner : Le sort s'acharne contre lui, ou encore du classique vaccin contre la grippe? puisqu'il s'agit, d'après C. Vandeloise (p. 139), de «la non-satisfaction de x à propos d'un processus impliqué par y». Va-t-on dire que « $\mathrm{x}$ n'est pas satisfait»? Admettons maintenant que des sujets inanimés puissent ne pas être satisfaits, n'y a-t-il pas quelque risque à construire pour un type d'emploi une règle d'usage qui n'a rien à voir avec ce qu'on a donné comme la valeur de base, c'est-à-dire les emplois spatiaux ? Il est clair que le rapprochement que va tenter de faire l'auteur entre les deux types d'emplois s'annonce délicat.

Mais, fidèle à son point de départ, il annonce (p. 139) qu'il va tenter «d'établir un parallélisme entre ces usages, fondé sur le mouvement ».

\section{Comparaisons entre usages spatiaux et axiologiques de contre}

4 On sait que le mouvement est un élément essentiel de la description de C. Vandeloise. I a toujours insisté sur le caractère dynamique des relations construites par contre ${ }^{18}$, c'est donc sur ce point que se focalise son attention. Le problème est évidemment de retrouver le mouvement dans les énoncés axiologiques. Comme il l'écrit lui-même, "Pour établir un parallèle fondé sur le mouvement entre ces usages (type « se défendre contre ») et les usages axiologiques de $x$ est contre $y$, il faut établir que, dans ce dernier cas :

a. $x$ n'est pas obligatoirement interprété comme un agent mobile

b. le site $y$ peut être considéré comme un élément mobile susceptible d'être arrêté par $x$ »

ce qui débouche à propos de l'énoncé :

(80) Ferdinand est contre les petits pois.

sur le grand moment d'humour et de glose suivant (p. 141) : 
«Cette phrase ne serait pas pertinente s'il n'existait pas dans le contexte quelqu'un ou quelque chose qui est pour les petits pois. Je n'aime pas les petits pois et je ne voudrais certainement pas en manger ce soir mais, tant que je ne suis pas menacé, je n'ai aucune raison de dire que je suis contre les petits pois. Il est donc raisonnable d'interpréter les petits pois dans la phrase (80) comme une menace qui s'avance vers Ferdinand (cnqs) qui souhaite éviter cette approche ${ }^{19}$. »

J'arrête, là, la reprise de la recherche pour le moins incertaine du mouvement dans les emplois axiologiques et j'en viens directement au tableau (p. 142) résumant les parallélismes dégagés par l'auteur et que je reproduis ci-dessous :

\begin{tabular}{|c|c|c|}
\cline { 2 - 3 } \multicolumn{1}{c|}{} & Spatial & Axiologique \\
\hline $\begin{array}{c}\text { Entité-sujet immobile ou } \\
\text { affectée } \\
\text { Site mobile }\end{array}$ & $\begin{array}{c}\text { Le mur protège contre les } \\
\text { voleurs }\end{array}$ & $\begin{array}{c}\text { Ferdinand est contre la } \\
\text { guerre }\end{array}$ \\
\hline $\begin{array}{c}\text { Entité-sujet agent } \\
\text { Site immobile }\end{array}$ & $\begin{array}{c}\text { Ferdinand jette des pierres } \\
\text { contre le mur }\end{array}$ & $\begin{array}{c}\text { Ferdinand écrit des livres } \\
\text { contre la guerre }\end{array}$ \\
\hline $\begin{array}{c}\text { Entité-sujet mobile } \\
\text { Site immobile }\end{array}$ & $\begin{array}{c}\text { Ferdinand bute contre une } \\
\text { pierre }\end{array}$ & $\begin{array}{c}\text { La guerre contre les } \\
\text { principes de Ferdinand }\end{array}$ \\
\hline & L'échelle est contre le mur & 0 \\
\cline { 2 - 3 }
\end{tabular}
spatiaux est donc un échec. On peut considérer, cependant comme le fait $\mathrm{C}$. Vandeloise, qu'il ne s'agit là que d'un défaut local, mais que, sur le fond, la théorie spatiale est toujours pertinente, dans la version défendue depuis 1986. C'est donc ce qu'il va tenter de montrer en proposant de réunir l'ensemble des emplois sous la notion de «rencontre atypique ». On voit bien, et l'auteur a passé beaucoup de temps sur ce point, que la démonstration repose sur la réalité du mouvement dans les emplois axiologiques (puisqu'elle est - à peu près - établie pour les emplois spatiaux). Si cela n'est pas montré, c'est le concept même de « rencontre » qui est invalidé. Il se trouve que le tableau comparatif proposé par l'auteur lui-même nous fait douter sérieusement des assises de son raisonnement. Comment croire en effet qu'à la ligne 1, dans Ferdinand est contre la guerre, le site (la guerre) est mobile tandis qu'à la ligne 2 dans Ferdinand écrit des livres contre la guerre, le site (la guerre) est immobile ${ }^{20}$ ? 


\section{Conclusion}

l'ensemble des emplois de la préposition. Mais il échoue à le faire pour les axiologiques qui ne mobilisent pas la notion de mouvement. On peut donc s'étonner de voir l'auteur affirmer, dans sa conclusion, l'intérêt du concept spatial, alors qu'il ne l'a appliqué, avec un succès, relatif, ainsi qu'il l'a écrit lui-même, qu'à une partie des emplois de la préposition : les emplois spatiaux.

\section{NOTES}

1. «Contre La rencontre atypique " dans Recherches linguistiques, 27, 2005, «Le mouvement dans la langue et la metalangue », P. Dendale éd., Université de Metz, pp. 121-150.

2. Avec en particulier les relations porteur/porté et contenant/contenu mobilisées pour décrire les prépositions sur et dans.

3. Il ne fait pas que cela, bien sûr, puisqu'il ramène les emplois de sur à la relation Porteur/Porté qui en est selon lui le prototype sémantique, mais les schémas ou les dessins sont, tout au long du livre, des éléments essentiels de l'analyse.

4. C'est une des caractéristiques de cette approche de caractériser les prépositions autres que à et de comme des "prépositions spatiales » tandis qu'ailleurs l'auteur peut parler des «emplois spatiaux » des mêmes prépositions, mais sans qu'il justifie explicitement cette caractérisation, qui relève en rélatité des axiomes de la théorie localiste.

5. On pourrait trouver gênant que contre soit définir par un mot de la même famille, mais $C$. Vandeloise semble s'en accommoder. Il l'évoque lui-même, dans une note $(5: 122)$ qui finalement ne dit rien sur le fond: "Ce n'est que longtemps après avoir introduit la rencontre dans mon analyse que je me suis rendu compte de la parenté morphologique de ce mot avec contre».

6. cnqs est mis pour «c'est nous qui soulignons». 
7. Vandeloise ne dit pas si la notion de « rencontre " vaut ou non pour d'autres prépositions. On peut le supposer si l'on pense à des exemples comme: La voiture est rentrée à toute allure dans la cité. J'ai envoyé une lettre à Florence. Allons au salon, etc.

8. L'auteur écrit (p. 216) «Cette section (2.1) réunit les trois types d'usages spatiaux les plus fréquents de la préposition contre ", tandis qu'il ne donne qu'un seul énoncé dans la dernière section (2.4) : (46) L'école est (tout) contre la mairie.

9. Comme pour la plupart des événements-types dans les articles sur contre, celui-ci trouve son origine dans l'article de M>.-T. Scepping (1991), qui le formulait ainsi / Sa maison est contre la mienne tout en faisant remarque que la réciproque: Ma maison est contre la sienne était possible.

10. Le mot section désigne à la fois des sous-parties de l'article et les classes d'énoncés qui y sont traités successivement.

11. Cette caractéristique des énoncés axiologiques est étudiée dans P. Péroz (2002). Elle joue un rôle important dans la caractérisation unitaire de la préposition proposée par l'auteur.

12. La notion de "contact» est au centre du travail de P. Dendale (2002), qui propose un «traitement unifié » des valeurs de la préposition dans un article que cite C. Vandeloise dans sa bibliographie.

13. M.-T. Schepping (1991 : 229) donne cet énoncé avec deux points d'interrogation, considérant qu'on trouvera plus naturellement : L'échelle est appuyée contre le mur.

14. "Cette interprétation est rendu plus facile par le fait que les soldats et Ferdinand sont capables de se déplacer. La règle Cs peut donc s'appliquer au mouvement qui a conduit aux situations décrites par les phrases (44) et (45)».

15. Il y avait évidemment la solution de faire appel à un observateur externe susceptible d'être à l'origine du rapprochement et donc du mouvement entre les deux entités mais C. Vandeloise, qui tient à la notion de « rencontre élémentaire » - hypothèse localiste oblige - et donc entre deux termes seulement, a exclu cette possibilité, comme on l'a vu, au début de son article (p. 124). Voir supra au $\S 1$.

16. On pourrait faire remarquer qu'il existe au moins un cas où cela est possible dans le domaine spatial, c'est le cas du belgicisme « pousser (ou laisser) la porte contre».

17. A propos du choix de ce mot, C. Vandeloise rédige la note suivante $(17: 137):$ « Le primitif le plus proche de satisfaire dans la liste proposée par Wierznicka (1993 : 17) est vouloir ».

18. Sur ce point se rapporter à Vandeloise.

19. C'est le vocabulaire employé par le linguiste (" une menace qui avance ", "éviter cette approche ») qui induit l'idée d'un mouvement. En réalité, ce que souhaite le locuteur c'est la non actualisation d'un procès constuit comme possible en Sito. Il n'est donc pas nécessaire de faire intervenir la notion de «mouvement » ou de « rencontre » pour décrire ce type d'énoncé (sur ce point, cf. Péroz 2003 136).),

20. Il ne s'agit évidemment pas d'une erreur de frappe. Les analyses correspondantes (et contradictoires) sont faite aux pages 140 et 141 de l'article. 


\section{RÉSUMÉS}

Pierre Péroz montre qu'une appréhension d'abord spatiale de la préposition, dont dériveraient tous les autres emplois, s'avère insuffisante et inappropriée pour rendre compte de ses (im)possibilités distributionnelles, ce qu'illustre l'exemple de contre.

\section{AUTEUR}

\section{PIERRE PÉROZ}

Université de Metz / CELTED 